Bier DM, German JB, Lönnerdal B (eds): Personalized Nutrition for the Diverse Needs of Infants and Children. Nestlé Nutr Workshop Ser Pediatr Program, vol 62, pp 173-188,

Nestec Ltd., Vevey/S. Karger AG, Basel, (C) 2008.

\title{
Personalized Care of Pediatric Cancer Patients
}

\author{
Karen Rabin, Tsz-Kwong Man, Ching C. Lau
}

Texas Children's Cancer Center, Baylor College of Medicine, Houston, TX, USA

\begin{abstract}
One of the great success stories of clinical oncology is the improvement in the cure rates of pediatric acute lymphoblastic leukemia (ALL) from around 10\% in the 1960s to nearly $90 \%$ today. The primary factor responsible for this remarkable improvement is the personalization of treatment, with stratification of patients based on both disease and host characteristics in order to optimize therapy. While age, WBC, and immunophenotype provide a rudimentary system for classification of ALL, molecular factors are playing an increasingly important role in further individualization of ALL therapy. Such riskbased stratification strategies are also increasingly being used in the treatment of children with solid tumors. In addition, genomic technologies are now being used to identify new molecular markers or signatures for both diagnostic and prognostic purposes. Recently we reported the analysis of pediatric osteosarcoma by expression profiling in an attempt to identify a molecular signature that could predict the chemoresistance of a tumor before treatment is initiated. We identified a 45-gene signature that discriminates between good and poor responders to chemotherapy in osteosarcoma. Using this classifier, we can predict with $100 \%$ accuracy the chemoresponse of osteosarcoma patients prior to the initiation of treatment. These encouraging results suggest that the genomic approach will revolutionize the diagnosis and prognosis of pediatric cancer patients and improve their outcome through predictive, personalized care.
\end{abstract}

Copyright @ 2008 Nestec Ltd., Vevey/S. Karger AG, Basel

\section{Introduction}

It has always been the goal in oncology to customize therapy for cancer patients in order to optimize long-term survival while minimizing the side effects of therapy. This is particularly important in the treatment of children with cancer because the potential side effects of therapy on the rest of the patient's rapidly growing body could be unacceptable or irreversible. Such personalization of treatment is usually based on the assessment of the aggressiveness of the cancer 
as well as the potential response of the cancer and the rest of the body to treatment. The former assessment is traditionally based on the extent of the spread of the disease at diagnosis as well as histologic subtypes within the same diagnostic group that are associated with poor outcome. The latter assessment is based on our previous observations of the response of a particular cancer type to standard therapy and the severity of toxicity patients had experienced. However, it has been difficult to predict the response to treatment or the side effects in a particular patient before therapy is initiated. Here, we will use pediatric acute lymphoblastic leukemia (ALL) and osteosarcoma to highlight the impact of personalized treatment in the clinical outcome of patients, and to illustrate how we have begun to augment the risk assessment of cancer patients by including novel molecular signatures identified by genomic technologies.

\section{Acute Lymphoblastic Leukemia}

ALL is the most common childhood cancer, with a peak incidence between 2 and 5 years of 4-5 per 100,000. Treatment of pediatric ALL is one of the great success stories of clinical oncology over the past several decades. ALL was a generally fatal diagnosis in the 1960 s, with cure rates around $10 \%$. In the decades that followed, the cure rate has steadily risen and today is approaching 90\% [1]. One of the primary factors responsible for this remarkable improvement is the personalization of treatment, with stratification of patients based on both host and disease characteristics in order to optimize therapy (table 1).

\section{Initial Classification}

The cornerstone of ALL therapy is stratification of patients into different risk groups based on a combination of clinical, laboratory, and molecular features, so that the type and intensity of therapy may be tailored appropriately. The initial classification as high versus standard risk is made on the basis of age and initial white blood count (WBC), two powerful and independent prognostic factors. High risk is defined by age $<1$ or $>10$ years, and/or initial $\mathrm{WBC}>50,000 / \mu \mathrm{l}[2]$. Immunophenotype also plays an important role in determining the choice of therapeutic regimen: T-cell ALL regimens differ somewhat from B-precursor ALL regimens based on the differential chemosensitivity of T-lineage lymphoblasts. Mature B-cell (Burkitt's) leukemia therapy is unique in its short duration and high intensity, tailored to the unique, aggressive biologic features of this subtype.

While age, WBC, and immunophenotype provide a rudimentary system for categorization of ALL, numerous other factors are playing an increasingly important role in further individualization of ALL therapy. 
Table 1. Factors with potential influence on ALL therapy

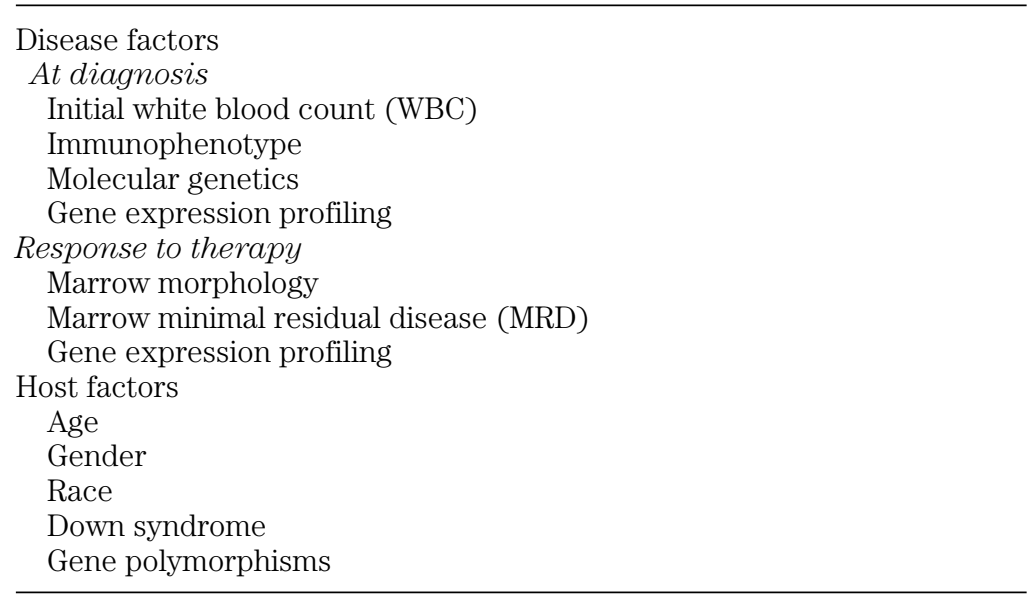

\section{Disease Factors}

\section{Molecular Genetics}

An understanding of molecular genetics has played a key role in optimizing therapy in pediatric ALL, defining low-risk subgroups which can be spared unnecessary toxicity, and high-risk subgroups which require more intensive therapy to increase the likelihood of cure. Approximately $75 \%$ of pediatric ALL cases bear a recurrent chromosomal abnormality with known prognostic relevance [3].

\section{Abnormalities of Chromosome Number (Ploidy)}

Hyperdiploid cases, with more than 46 chromosomes, constitute about $35 \%$ of pediatric ALL. Hyperdiploidy has an independent positive prognostic value, which was recently recognized as being attributable to specific trisomies, with the most favorable being chromosomes 4, 10, and 17, and not simply total chromosome number [4]. Hypodiploid cases, with fewer than 45 chromosomes, have significantly worse outcome than diploid or hyperdiploid ALL, with the worst outcome in near-haploid cases (24-28 chromosomes).

\section{Abnormalities of Chromosome Structure in ALL}

Chromosomal translocations are a cardinal feature of ALL. Most often the translocation brings a proto-oncogene into proximity with a T-cell receptor or immunoglobulin locus, causing overexpression of the intact gene; or genes at the translocation breakpoints, often transcription factors, may fuse to form a 
Table 2. Common chromosomal abnormalities in pediatric ALL

\begin{tabular}{llll}
\hline $\begin{array}{l}\text { Chromosomal } \\
\text { abnormality }\end{array}$ & $\begin{array}{l}\text { Genes } \\
\text { involved }\end{array}$ & $\begin{array}{l}\text { Frequency } \\
(\%)\end{array}$ & Prognostic impact \\
\hline $\mathrm{t}(12 ; 21)(\mathrm{p} 13 ; \mathrm{q} 22)$ & TEL-AML1 & 25 & Favorable \\
$9 \mathrm{p} 21$ deletion & p15, p16 & 10 & Uncertain \\
$\mathrm{t}(4 ; 11)(\mathrm{q} 21 ; \mathrm{q} 23)$ & MLL-AF4 & 8 & $\begin{array}{l}\text { Poor } \\
\mathrm{t}(1 ; 19)(\mathrm{q} 23 ; \mathrm{p} 13)\end{array}$ \\
E2A-PBX1 & 6 & $\begin{array}{l}\text { Formerly poor; negated by } \\
\text { intensive therapy }\end{array}$ \\
$\mathrm{t}(9 ; 22)(\mathrm{q} 34 ; \mathrm{q} 11)$ & BCR-ABL & 3 & Poor \\
$\mathrm{t}(8 ; 14)(\mathrm{q} 24 ; \mathrm{q} 32)$ & MYC, IgH & 2 & Poor; partly negated by \\
& & & Burkitt's-directed therapy \\
\hline
\end{tabular}

new, chimeric protein with oncogenic effects [5]. The most common prognostically significant chromosomal translocations associated with pediatric ALL are listed in table 2.

The TEL-AML1 fusion protein formed by the t(12;21)(p13;q22) translocation occurs in approximately $25 \%$ of childhood ALL, making it the most frequent abnormality in childhood ALL. TEL-AML1 is generally associated with a more favorable prognosis, although late relapses are relatively frequent. However, relapsed TEL-AML1+ ALL generally demonstrates an excellent chemosensitivity and salvage rate.

The E2A-PBX1 fusion protein, associated with the $t(1 ; 19)(q 23 ; p 13)$ translocation, is the second most common translocation in pediatric ALL, occurring in approximately $6 \%$ of pediatric pre-B ALL. The E2A-PBX1 fusion protein tends to be associated with other known high-risk factors. In early studies it was found to have an independent adverse prognostic impact, but on modern intensive treatment regimens, survival is equivalent.

The $\mathrm{t}(9 ; 22)$ translocation, known as the Philadelphia chromosome (Ph), is an essential criterion in the diagnosis of chronic myeloid leukemia (CML) and the most frequent abnormality in adult ALL, but occurs in only about $3 \%$ of pediatric ALL. Ph is an adverse prognostic marker, with significantly lower induction rates, more frequent and earlier relapse, and poorer overall survival. Allogeneic stem cell transplant has generally been regarded as the only curative therapy, and is generally recommended in first complete remission. Treatment of CML and Ph+ ALL was revolutionized by the advent of imatinib mesylate, also known as Gleevec, in 2001 [6]. Imatinib, a selective tyrosine kinase inhibitor, was the first molecularly targeted therapy to attain largescale clinical success, fulfilling the goals of antitumor selectivity and low systemic toxicity. Despite its success, it has not been effective as a single agent due to the rapid development of resistance, and allogeneic stem cell transplant in first complete remission remains the optimal curative therapy. 
MLL gene rearrangements occur in $8 \%$ of pediatric ALL and $60-70 \%$ of infant ALL. Over 40 MLL fusion partners have been identified, but the MLLAF4 fusion formed by $\mathrm{t}(4 ; 11)$ is the most common in ALL. MLL rearrangement in infant ALL is a poor prognostic factor, associated with frequent early relapse. Gene expression studies have revealed that the receptor tyrosine kinase FLT3 is highly expressed in MLL-rearranged ALL, and targeted FLT3 inhibitor therapy for MLL-rearranged ALL is an area of active investigation $[7,8]$.

The $\mathrm{t}(8 ; 14)$ (q24;q32) translocation places the c-MYC oncogene under control of the immunoglobulin heavy chain promoter, resulting in constitutive cMYC overexpression. MYC dysregulation is an essential feature of mature B cell, also known as Burkitt's lymphoma and leukemia. Outcomes have markedly improved since the recognition that this molecular abnormality defines a group of diseases that require specific, short and highly intensive chemotherapy $[9,10]$.

Microarray-based analysis of gene expression patterns is emerging as an additional powerful tool for defining ALL subgroups. Expression arrays have identified highly overexpressed genes which are candidate targets for development of new therapies, such as FLT3 in MLL [7]. Microarray analysis has also characterized gene expression changes in response to particular chemotherapeutic agents $[11,12]$ and identified expression patterns associated with chemotherapy sensitivity versus resistance [13].

\section{Response to Therapy}

Early response to therapy is a powerful, independent predictor of prognosis, and is used on many current protocols as a determinant of subsequent therapy. Response to therapy is assessed both by marrow morphology, typically after 1-2 weeks of induction therapy, and by minimal residual disease (MRD) assessment, typically at the conclusion of the month-long induction regimen. MRD, based on flow cytometry or PCR-based assays, is far more sensitive than morphology and has been shown to identify patients at risk of ultimate morphologic relapse [14]. Conversely, rapid achievement of MRD negativity before the end of induction identifies a favorable prognosis group with a high probability of cure, who might be spared the adverse effects of high-intensity regimens.

\section{Host Factors}

Gender is a basic host factor long recognized as affecting prognosis, with males having a survival disadvantage, at least in part due to the testicles being a sanctuary site to chemotherapy and therefore a frequent site of relapse. In the current US Children's Oncology Group protocol, males are treated for a full year longer than females to compensate for the gender discrepancy in prognosis. 
Race also has an impact on prognosis, with African-Americans and, to a lesser extent, Hispanics doing somewhat more poorly than Caucasians [15]. This appears to be due in part to more frequent high-risk molecular and clinical features, and less frequent low-risk features among these ethnicities. However, even within each risk category, African-Americans and Hispanics seem to do somewhat more poorly.

Pharmacogenetic factors are increasingly recognized as important determinants of prognosis in oncology. A prime example is the effect of host polymorphisms in thiopurine methyltransferase (TPMT), a key enzyme involved in inactivation of mercaptopurine, a mainstay of ALL maintenance chemotherapy [16]. Several gene polymorphisms cause decreased TPMT activity and hence increased exposure to active drug, which leads to both a greater antileukemic effect and greater systemic toxicity, including myelosuppression and increased long-term risk of second malignancy. It is now standard practice to perform TPMT genotyping either in all patients prior to therapy, or in those patients exhibiting severe myelosuppression, to identify those who require mercaptopurine dose modification.

Patients with Down syndrome constitute another group in which pharmacogenetic factors play an important role in modulating therapy. Methotrexate has long been noted to cause increased toxicity in Down syndrome patients, apparently due to an extra copy of the reduced folate carrier gene on chromosome 21, which is responsible for intracellular transport of methotrexate, leading to higher intracellular methotrexate levels at a given dose level compared to non-Down syndrome patients, and hence increased somatic toxicity. To minimize toxicity, a substantial reduction in methotrexate dosing in Down syndrome patients has been adopted in many current protocols.

Glutathione S-transferases, which inactivate many ALL chemotherapeutic agents, harbor frequent polymorphisms which have also not surprisingly been associated with increases both in chemotherapeutic efficacy and in toxicities such as second malignancies [17]. Polymorphisms in thymidylate synthase, a target of methotrexate, have been associated with an increased risk of relapse. The effects of these gene polymorphisms vary somewhat across studies, suggesting that effects are likely influenced by treatment and other factors.

\section{Osteosarcoma}

We have much experience customizing therapy for leukemia patients based on risk assessment as described above. However, such therapeutic strategies have not been as well developed in the treatment of solid tumors until very recently because of the lack of validated prognostic makers. In the past few years, we and others have tested the feasibility of using comprehensive molecular profiling technologies to identify biomarkers for both diagnostic and prognostic purposes. Using osteosarcoma as an example, we will 


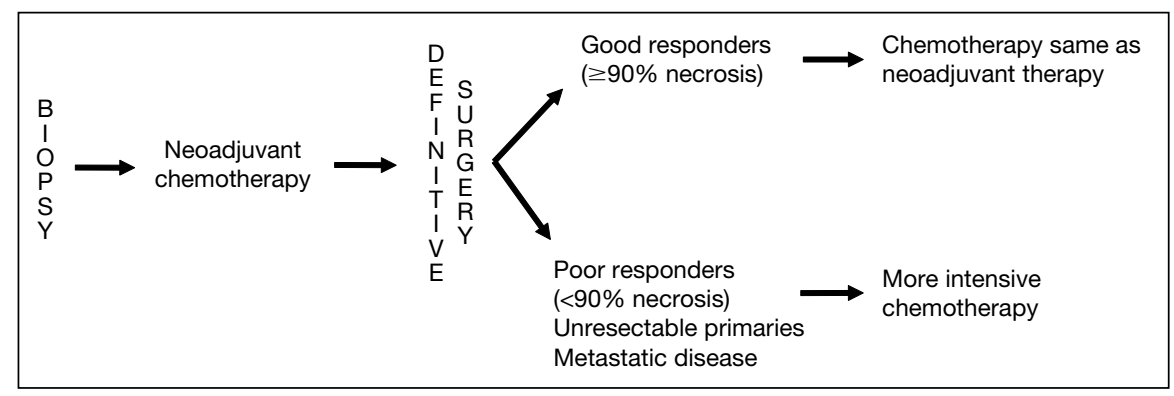

Fig. 1. Treatment scheme for osteosarcoma. All patients with localized disease and good response to preoperative chemotherapy will receive the same chemotherapeutic agents in postoperative chemotherapy. Poor risk patients including the poor responders and those who have unresectable primary tumors or metastatic disease will receive more intensive chemotherapy as postoperative therapy.

illustrate how these biomarkers have been identified and validated. One such application is the use of a multi-gene signature to predict the response to chemotherapy at the time of diagnosis prior to the initiation of therapy.

Osteosarcoma is the most common malignant bone tumor in children and accounts for approximately $60 \%$ of malignant bone tumors diagnosed in the first 2 decades of life [18]. After the diagnosis is made by an initial biopsy (IB), standard treatment involves the use of multi-agent chemotherapy, definitive surgery (DS) of the primary tumor, and postoperative chemotherapy (fig. 1). At the time of DS, the resected tumor specimen is assessed for the degree of necrosis which is used to guide the choice of postoperative chemotherapy. The degree of necrosis at the time of DS is a reliable and only significant prognostic factor in patients with non-metastatic disease. Patients whose tumors display $\geq 90 \%$ necrosis (good or favorable response) have an excellent prognosis and continue to receive chemotherapy similar to the preoperative regimen. Patients whose tumors display $<90 \%$ necrosis (poor or unfavorable response) have a much higher risk of relapse and poor outcome even after complete resection of the primary tumor [19]. To improve the outcome of the poor responders, attempts are usually made to use postoperative chemotherapy regimens that are different from the preoperative regimen by the addition or replacement of a chemotherapeutic agent. In the past such attempts have been unsuccessful $[18,20]$, partly because the degree of necrosis is known only after $8-10$ weeks of preoperative therapy. It is possible that resistant tumor cells have additional time to either metastasize to the lungs or to evolve further during the period when ineffective pre-operative chemotherapy is given. Therefore, at the time of initial diagnosis, there is a need to identify the patients who are likely to have a poor response to standard 
preoperative therapy and therefore a poor outcome eventually. Therapies tailored to improve the outcome of those patients identified at the time of diagnosis to have a poor outcome can then be instituted at the outset when the chance for success is potentially higher. Although a number of other prognostic factors have been proposed for predicting the long-term outcome of osteosarcoma patients, most are still controversial or have not been tested in large prospective studies [21-28].

\section{Expression Profiling of Osteosarcoma}

Recently we reported the analysis of 34 pediatric osteosarcoma samples by expression profiling [29]. With the goal of identifying molecular signatures that can predict the chemoresistance of osteosarcoma, we first attempted to determine the expression profiles of resistant versus sensitive osteosarcoma cells. We hypothesized that the DS samples from the poor responders (PR) should be enriched for resistant tumor cells. Using expression profiles from DS samples would therefore enhance the sensitivity and power to detect the difference between chemosensitive and resistant cell populations as compared to using IB samples in which resistant cells may only be present as a small fraction. Therefore, we predicted that using DS samples would increase the chance of identifying a molecular signature of chemoresistance and, if this signature is valid, it could be used to identify the good responders (GR) and PR in the IB samples. To test this hypothesis, we examined if we could classify GR and PR using DS specimens only. We divided the DS samples from 20 patients into 2 groups, GR $(n=7)$ and PR $(n=13)$. We first identified a set of 45 predictor genes that could discriminate the two classes (GR and PR) in the DS samples using a 2-sample $t$ test with a significant cutoff $(p=0.005)$. Figure 2 shows the relative expressions of these 45 predictor genes in GR and PR. Most of these genes (91\%) were overexpressed in PR specimens.

Various supervised classification algorithms, Compound Covariate Predictor, K-Nearest Neighbor, Nearest Centroid, Support Vector Machine (SVM), and Linear Discriminant Analysis, , were then applied to the training set to test if they could classify GR and PR using a p value of 0.005 . The Leave-One-Out Cross-Validation (LOOCV) method was used to test the robustness of each classifier in the training set (table 3). The correct classification rates of LOOCV using these algorithms were 65-70\%. Among the 6 algorithms, SVM had one of the best performances (70\% correct classification). Three GR and three PR were misclassified using SVM. Two of the GR (No. 300 and 394) that were misclassified as PR by the SVM classifier developed recurrent disease 11 and 9 months, respectively, after completion of therapy. This suggests that there may be some residual resistant cells in the DS specimens of these two cases that were recognized by the algorithm based on the predictor gene set. One of the PR (No. 680) that was misclassified as a GR by the SVM classifier remains free of disease after 30 months of follow-up. 


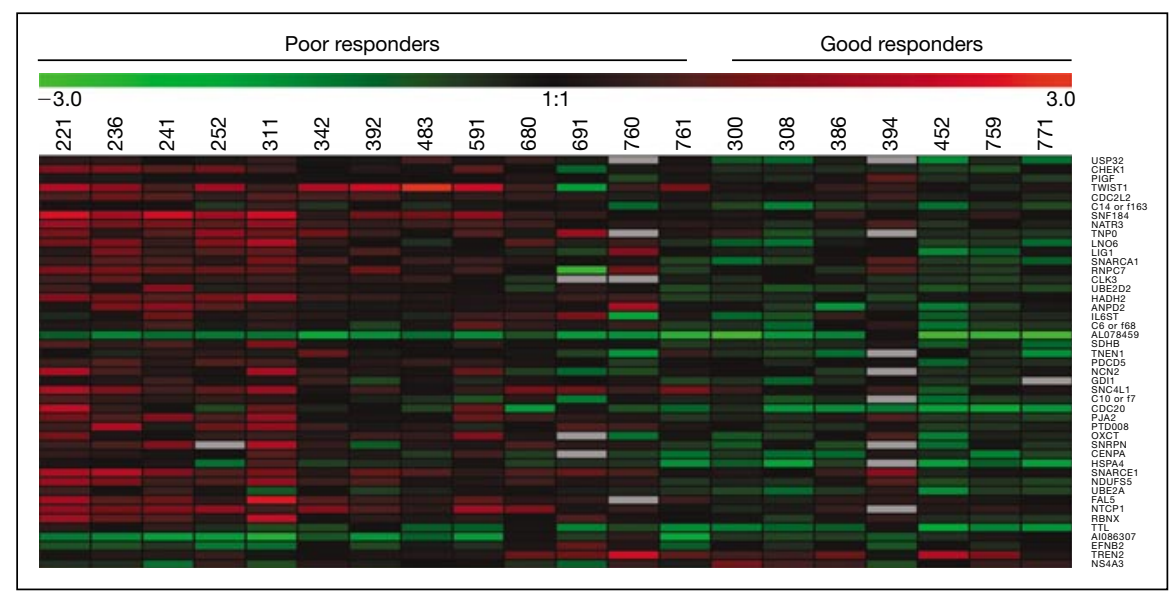

Fig. 2. The 45 predictor genes in the chemoresistance signature were selected based on a 2-sample t test to distinguish between good and poor responders in 20 definitive surgery samples at a p value of 0.005 . Forty-one genes were overexpressed in poor responders, while only 4 genes were overexpressed in good responders. The color scale represents log 2 expression ratios of the genes.

The other misclassified PR (No. 761) had 86\% necrosis, which is very close to our cutoff for good response.

\section{Use of Multi-Gene Classifier to Predict Response to Preoperative}

Chemotherapy in $I B$

To test the SVM classifier, we divided our 14 IB samples into two groups. The first group consisted of six samples, which had corresponding DS samples included in the training set (paired samples). Using these six cases, we attempted to verify that our classifier built from DS samples could predict the chemoresistance of the corresponding IB samples based on the hypothesis that the molecular signature of chemoresistance as recognized in DS samples was already present in the IB at the time of diagnosis. The second group consisted of eight IB samples that did not have matched DS samples included in the training set, thus representing a totally independent set of samples that had not been used to build the classifier.

The SVM classifier misclassified one sample (of 6) in the first group of paired samples, with a correct classification rate of $83 \%$ (95\% CI 36, 100; table 4). The only misclassified sample was from a patient (No. 410) who was classified as a GR based on histologic response but was predicted to be a PR by the multi-gene classifier. Interestingly, this patient initially presented with localized disease but eventually developed recurrent disease in the lungs 25 months after completion of therapy, suggesting that there were resistant cells 
Table 3. Leave one out cross-validation of 20 definitive surgery osteosarcoma samples

\begin{tabular}{|c|c|c|c|c|c|c|c|}
\hline \multirow[t]{2}{*}{ Tumor ID } & \multirow[t]{2}{*}{$\begin{array}{l}\text { Histologic } \\
\text { response }\end{array}$} & \multicolumn{6}{|c|}{$\begin{array}{l}\text { Concordance of classification with histological } \\
\text { response }\end{array}$} \\
\hline & & $\mathrm{CCP}$ & LDA & $1-\mathrm{NN}$ & $3-\mathrm{NN}$ & $\mathrm{NC}$ & SVM \\
\hline 300 & GR & No & No & No & No & No & No \\
\hline 308 & GR & Yes & Yes & Yes & Yes & Yes & Yes \\
\hline 386 & GR & No & No & No & No & No & No \\
\hline 394 & GR & No & No & No & No & No & No \\
\hline 452 & GR & Yes & Yes & Yes & Yes & Yes & Yes \\
\hline 759 & GR & Yes & Yes & Yes & Yes & Yes & Yes \\
\hline 771 & GR & Yes & Yes & Yes & Yes & Yes & Yes \\
\hline 221 & PR & Yes & Yes & Yes & Yes & Yes & Yes \\
\hline 236 & PR & Yes & Yes & Yes & Yes & Yes & Yes \\
\hline 241 & PR & Yes & Yes & Yes & Yes & Yes & Yes \\
\hline 252 & PR & Yes & Yes & Yes & Yes & Yes & Yes \\
\hline 311 & PR & Yes & Yes & Yes & Yes & Yes & Yes \\
\hline 342 & PR & Yes & Yes & Yes & Yes & Yes & Yes \\
\hline 392 & PR & Yes & Yes & Yes & Yes & Yes & Yes \\
\hline 483 & PR & Yes & Yes & Yes & Yes & Yes & Yes \\
\hline 591 & PR & Yes & Yes & Yes & Yes & Yes & Yes \\
\hline 680 & PR & No & No & No & No & No & No \\
\hline 691 & PR & No & No & No & No & No & No \\
\hline 760 & PR & No & Yes & Yes & Yes & No & Yes \\
\hline 761 & PR & No & No & No & No & No & No \\
\hline $\begin{array}{l}\text { \% Correctly } \\
\text { classified }\end{array}$ & & 65 & 70 & 70 & 70 & 65 & 70 \\
\hline
\end{tabular}

$\mathrm{CCP}=$ Compound Covariate Predictor; LDA = Linear Discriminant analysis; $\mathrm{NN}=$ Nearest Neighbor; NC $=$ Nearest Centroid; SVM = Support Vector Machine; $\mathrm{GR}=$ good responders; $\mathrm{PR}=$ poor responders; Yes $=$ classification by the algorithm was correct; No = classification was wrong.

present in the IB that were recognized by the multi-gene classifier, and presumably these resistant cells metastasized to the lungs prior to DS and subsequently gave rise to the recurrent tumor. Ironically the multi-gene predictor classified this patient's DS sample (No. 452) as a GR, implying that either the DS sample used in our analysis was not representative of the primary tumor in that it did not contain the resistant cells, or that the resistant cells had already metastasized before DS and therefore were no longer detectable in the primary tumor.

In the second group of independent IB samples, the classifier correctly predicted 8 of 8 of the samples (100\% correct, $95 \%$ CI 63, 100). These 8 samples included $5 \mathrm{PR}$ and $3 \mathrm{GR}$. These results further indicate that the gene expression signature of the resistant cells in the DS samples was already 
Table 4. Classification of initial biopsy samples using a support vector machine classifier

\begin{tabular}{llll}
\hline Tumor ID & $\begin{array}{l}\text { Histologic } \\
\text { response }\end{array}$ & \multicolumn{2}{l}{$\begin{array}{l}\text { Concordance with histologic } \\
\text { response }\end{array}$} \\
\cline { 3 - 4 } & & paired & independent \\
\hline 410 & GR & No & \\
197 & PR & Yes & \\
207 & PR & Yes & \\
278 & GR & Yes & \\
289 & PR & Yes & Yes \\
345 & GR & Yes & Yes \\
204 & PR & & Yes \\
274 & PR & & Yes \\
299 & GR & & Yes \\
464 & PR & & Yes \\
479 & PR & & Yes \\
481 & PR & & \\
545 & GR & & \\
654 & GR & & \\
\hline GR $=$ Good & responder; PR = poor responder. & \\
\hline
\end{tabular}

present in the IB samples at the time of diagnosis. Our result is consistent with the notion proposed by Ramaswamy et al. [30] that the metastatic signature of metastatic tumors is already present in the primary tumor. The high accuracy of our multi-gene classifier to identify GR and PR from two separate groups of IB samples suggests that response to chemotherapy can potentially be predicted at the time of diagnosis. However, due to the limited number of samples used in the study, the classifier and chemoresistant signature needs to be validated in a larger multi-institutional study. If validated, this can significantly impact the design of future therapeutic studies of osteosarcoma, in which intensified therapy could be given at the time of diagnosis to those patients who are predicted to be PR to standard therapy in order to improve their outcome.

\section{Conclusion}

As illustrated by the examples of pediatric ALL and osteosarcoma, it is apparent that molecular classification will be the driving force in the further development of individualized therapy for cancer patients. We believe that molecular profiling will provide important clues regarding critical pathways 
that cancer cells are dependent on to maintain their malignant phenotype. Such pathways will be ideal therapeutic targets for tumor-specific therapies. An equally important aspect of this goal will involve the continuation of ongoing efforts to cautiously decrease the intensity of potentially toxic therapies in order to reduce the morbidity of treatment in tumors that have particularly favorable risk factors based on genomic profiling. In conclusion, genomic profiling analysis offers an exciting possibility for refining the diagnosis, stratification and therapy of pediatric cancers. It is reasonable to imagine that in the near future, predictive individualized care based on molecular classification and targeted therapy will become a reality for children with cancer.

\section{Acknowledgement}

This work was supported by NIH grants CA88126, CA97874, CA109467, and CA114757 as well as grants from the John S. Dunn Research Foundation and the Robert J. Kleberg, Jr. and Helen C. Kleberg Foundation, Gillson Longenbaugh Foundation and Cancer Fighters of Houston, Inc.

\section{References}

$\checkmark 1$ Pui CH, Evans WE: Treatment of acute lymphoblastic leukemia. N Engl J Med 2006;354: 166-178.

-2 Smith M, Arthur D, Camitta B, et al: Uniform approach to risk classification and treatment assignment for children with acute lymphoblastic leukemia. J Clin Oncol 1996;14:18-24.

-3 Armstrong SA, Look AT: Molecular genetics of acute lymphoblastic leukemia. J Clin Oncol 2005;23:6306-6315.

-4 Sutcliffe MJ, Shuster JJ, Sather HN, et al: High concordance from independent studies by the Children's Cancer Group (CCG) and Pediatric Oncology Group (POG) associating favorable prognosis with combined trisomies 4, 10, and 17 in children with NCI Standard-Risk B-precursor Acute Lymphoblastic Leukemia: a Children's Oncology Group (COG) initiative. Leukemia 2005;19:734-740.

5 Rabbitts TH: Chromosomal translocations in human cancer. Nature 1994;372:143-149.

-6 Druker BJ, Sawyers CL, Kantarjian H, et al: Activity of a specific inhibitor of the BCR-ABL tyrosine kinase in the blast crisis of chronic myeloid leukemia and acute lymphoblastic leukemia with the Philadelphia chromosome. N Engl J Med 2001;344:1038-1042.

7 Armstrong SA, Kung AL, Mabon ME, et al: Inhibition of FLT3 in MLL. Validation of a therapeutic target identified by gene expression based classification. Cancer Cell 2003;3:173-183.

$>8$ Stam RW, den Boer ML, Schneider P, et al: Targeting FLT3 in primary MLL-gene-rearranged infant acute lymphoblastic leukemia. Blood 2005;106:2484-2490.

-9 Cairo MS, Sposto R, Perkins SL, et al: Burkitt's and Burkitt-like lymphoma in children and adolescents: a review of the Children's Cancer Group experience. Br J Haematol 2003;120: $660-670$.

-10 Blum KA, Lozanski G, Byrd JC: Adult Burkitt leukemia and lymphoma. Blood 2004;104: 3009-3020.

11 Cheok MH, Yang W, Pui CH, et al: Treatment-specific changes in gene expression discriminate in vivo drug response in human leukemia cells. Nat Genet 2003;34:85-90.

12 Zaza G, Cheok M, Yang W, et al: Gene expression and thioguanine nucleotide disposition in acute lymphoblastic leukemia after in vivo mercaptopurine treatment. Blood 2005;106:1778-1785.

13 Holleman A, Cheok MH, den Boer ML, et al: Gene-expression patterns in drug-resistant acute lymphoblastic leukemia cells and response to treatment. N Engl J Med 2004;351:533-542. 
14 Cave H, van der Werff ten Bosch, Suciu S, et al: Clinical significance of minimal residual disease in childhood acute lymphoblastic leukemia. European Organization for Research and Treatment of Cancer - Childhood Leukemia Cooperative Group. N Engl J Med 1998;339: 591-598.

15 Pollock BH, DeBaun MR, Camitta BM, et al: Racial differences in the survival of childhood B-precursor acute lymphoblastic leukemia: a Pediatric Oncology Group Study. J Clin Oncol 2000;18:813-823.

16 Relling MV, Dervieux T: Pharmacogenetics and cancer therapy. Nat Rev Cancer 2001;1: 99-108.

$\checkmark 17$ Cheok MH, Evans WE: Acute lymphoblastic leukaemia: a model for the pharmacogenomics of cancer therapy. Nat Rev Cancer 2006;6:117-129.

18 Link MP, Gebhardt MC, Meyers PA: Osteosarcoma; in Pizzo P, Poplack D (eds): Principles and Practice of Pediatric Oncology, ed 5. Philadelphia, Lippincott-Williams \& Wilkins, 2006, pp 1074-1115.

19 Provisor AJ, Ettinger LJ, Nachman JB, et al: Treatment of nonmetastatic osteosarcoma of the extremity with preoperative and postoperative chemotherapy: a report from the Children's Cancer Group. J Clin Oncol 1997;15:76-84.

-20 Meyers PA, Heller G, Healey J, et al: Chemotherapy for nonmetastatic osteogenic sarcoma: the Memorial Sloan-Kettering experience. J Clin Oncol 1992;10:5-15.

21 Baldini N, Scotlandi K, Barbanti-Brodano G, et al: Expression of P-glycoprotein in high-grade osteosarcomas in relation to clinical outcome. N Engl J Med 1995;333:1380-1385.

-22 Bacci G, Longhi A, Ferrari S, et al: Prognostic significance of serum alkaline phosphatase in osteosarcoma of the extremity treated with neoadjuvant chemotherapy: recent experience at Rizzoli Institute. Oncol Rep 2002;9:171-175.

-23 Gorlick R, Huvos AG, Heller G, et al: Expression of HER2/erbB-2 correlates with survival in osteosarcoma. J Clin Oncol 1999;17:2781-2788.

24 Bielack SS, Kempf-Bielack B, Delling G, et al: Prognostic factors in high-grade osteosarcoma of the extremities or trunk: an analysis of 1,702 patients treated on neoadjuvant cooperative osteosarcoma study group protocols. J Clin Oncol 2002;20:776-790.

25 Davis AM, Bell RS, Goodwin PJ: Prognostic factors in osteosarcoma: a critical review. J Clin Oncol 1994;12:423-431.

$\checkmark 26$ Feugeas O, Guriec N, Babin-Boilletot A, et al: Loss of heterozygosity of the RB gene is a poor prognostic factor in patients with osteosarcoma. J Clin Oncol 1996;14:467-472.

$\checkmark 27$ Franzius C, Bielack S, Flege S, et al: Prognostic significance of (18)F-FDG and (99m)Tcmethylene diphosphonate uptake in primary osteosarcoma. J Nucl Med 2002;43:1012-1017.

-28 Ulaner GA, Huang HY, Otero J, et al: Absence of a telomere maintenance mechanism as a favorable prognostic factor in patients with osteosarcoma. Cancer Res 2003;63:1759-1763.

29 Man TK, Chintagumpala M, Visvanathan J, et al: Expression profiles of osteosarcoma that can predict response to chemotherapy. Cancer Res 2005;65:8142-8150.

30 Ramaswamy S, Ross KN, Lander ES, Golub TR: A molecular signature of metastasis in primary solid tumors. Nat Genet 2003;33:49-54.

\section{Discussion}

Dr. Haschke: This is probably a question which will be very difficult to answer. Using the genetic profiling of a tumor to look at aggressiveness and then designing tailor-made therapy, there is very little time so the profile is looked at only once because, for example in leukemia, it makes a difference whether therapy is started 5 days later or earlier. Could it be possible that the genetic profiling of the tumor could be changed if, for example, there were a few days time just to improve the nutritional status of the patient and see if the patient's immune response could be changed? Is this possible; are there animal models showing that the profiling of the tumor could be changed or modified? I know this is not possible because most tumors are not subjected to primary surgery but primarily to chemotherapy, so there is no time. But would it be possible or are there animal models showing that this profiling could change? 
Dr. Lau: Actually this type of study has been done in animals and initially the profiles were quite sensitive to all kinds of perturbations, but with the more recent improvements in the platforms these profiles are rather robust now. So it can actually be done at multiple time points in animal models, and nearly exactly the same results are obtained. It is true that with leukemia we actually have the luxury of doing that. A peripheral blood sample can be taken where there are leukemic blasts and at multiple time points, and very similar results are seen. With solid tumors we don't have that luxury.

Dr. Alhaffar: You mentioned a lot about chromosomal studies and gene locations in patients who had the disease. When you are doing a random study for chromosomes, especially for Down syndrome for instance, in pregnant women, and you find these dislocations and gene positions, what do you do between prediction and prevention? Do you force yourself to follow-up treatment? What do you do, you just found the gene?

Dr. Lau: That is one of the intrinsic problems of generating so much data. In clinical medicine we always say do not order a test if you are not ready to act on the data. This is precisely the problem. Some of the translocations can actually be found in children who do not have leukemia. For example the 12;21 translocation that I mentioned briefly was subsequently discovered and the same translocation can be detected in patients if you are able to retrieve the initial blood spots at the time of birth, the Guthrie spots. This suggests that the translocation has been there all along and that something else has triggered the development of these leukemic blasts into leukemia. Unfortunately or fortunately, we did controls for those studies and found that the same translocation can also be found in some Guthrie spots of otherwise normal children. So obviously this is not the only event that is required for leukemia to develop. We are still trying to find out exactly what other events need to happen in order for full-blown disease to develop. We are still at a rather infant stage in making use of these data in a clinical setting. So yes, we may be able to predict, but the prediction is not $100 \%$.

Dr. Björkstén: How do you handle the ethical aspects of knowing which osteosarcoma patients are going to have metastases yet not being able to do anything about it?

Dr. Lau: That is why the initial study has to be done in such a way that no information is conveyed back to the treating physician or the patient, because if we are only detecting certain events that may happen in the future but without any intervention strategies that creates an ethical challenge. We are now being challenged to find alternative therapy in association with the identification of these high-risk patients. We have created a problem for ourselves because we have the diagnostic capabilities but no therapeutic strategies available yet.

Dr. Waterland: You found a relatively small number of genes in which the expression on the array seems to predict responsiveness to osteosarcoma therapy. Have you done any type of gene ontology to look for related biochemical functions of those genes in order to predict what potential changes in therapy might improve the response?

Dr. Lau: Yes, we have started looking at that, and in fact there is a series of functional studies now validating the role of these genes in both osteosarcoma cells as well as normal osteoblast cells which we think are the progenitor cells for the tumor and some of them are very interesting. For example there is a gene called twist1 which turns out to be involved in the normal differentiation of osteoblasts, so immediately we know that it is relevant. But equally important, it was found to be associated with chemoresistance in other types of tumors; totally independent of our own findings, they are found in neuroblastoma, in colon cancer, in prostate cancer, etc. So we can land on highly relevant genes without any prior knowledge of where these genes could be.

Dr. Saavedra: This is really more of a comment and an opportunity to contrast what you just showed compared to what we were talking about previously. You have 
basically shown that there is at least one very good example where a predictive mechanism can be found using microarrays and very elegant genomics for a disease which is not that common. The specificity is greater than $90 \%$ and sensitivity is close to $100 \%$, looking at an oncologic disease. In the previous discussions we were looking at using family history to predict allergy risk, which has a sensitivity of less than $25 \%$ and the specificity is high as perhaps $70 \%$; because the majority of children who have a family history don't have allergy and most children who have allergy don't have a family history [1]. This is a challenge for us. We are using the most primitive kinds of predictive tools to analyze studies such as allergy, where we need to make important clinical decisions to curb this epidemic. This type of problem presents a challenge to us all. We need better predicitive tools or consider interventions that can be more broadly applied.

Dr. Berry: Regarding the altered genes that are expressed, do the proteomic analyses show primarily posttranslational modifications or altered levels?

Dr. Lau: So far the correlation between the genomic profiles and the proteomic profiles are not very good. This is not too surprising because it is a well-known fact in protein biochemistry that there is a very poor correlation between the RNA level and protein level, which is precisely what you were hinting at in the posttranslational modification, and now we have to worry about micro-RNA being involved, etc. But the interesting thing is that even though the levels do not correlate that well, they are actually pointing to the same pathways. In fact we actually believe that the pathway pinpointed by this profiling is more important than the individual genes or proteins because we now have the whole pathways that we can wrestle with rather than individual genes. Even though it is not the perfect correlation that everybody would like to see, I think they are pointing to the same critical pathways.

Dr. Berry: Are you struggling with being able to find internal standards to use to normalize the data within each tumor sample?

Dr. Lau: Actually it is getting less and less problematic because of the availability of the publicly available data, and we are now actually using what is out there to normalize our data. Fortunately to the result is very stable as I suggested earlier.

Dr. German: Would it be possible to use diet to enhance the diagnostic capabilities? Obviously the sensitivity and specificity are often high, but these are presumably on the background of children on varying diets. If it could actually be standardized and get that part relatively constant, would that improve your diagnostic capability?

Dr. Lau: That is a question that we all ask and that is why metabolomic profiling is being advocated and nobody can predict where it will be going. I always worry about that myself; what is the impact of the nutritional status of these patients, prior to presentation, that might affect the profiling results. We haven't done that kind of control studies yet.

Dr. Waterland: In your introduction you mentioned the potential to do some profiling on the genomic level and I am just wondering whether you eventually see the potential to do testing at birth using comparative genomic hybridization to look for duplications, deletions, the sort of thing that might eventually predict which individuals might be at risk for these types of diseases?

Dr. Lau: That is actually in planning right now. We are trying to retrieve a lot of the Guthrie spots in the State of Texas which are stored in Austin and repeat the studies and try to test that particular hypothesis. But the other thing I need to mention other than the SNP polymorphism, we now also worry about the copy number variation polymorphism as well. Some of you may or may not realize that the normal genome does not contain two copies of every gene. Close to 7,000 regions of the normal human genome have been identified that have copy numbers other than 2 , and many of them actually contain known genes. Many of these genes actually are the genes that code 


\section{Rabin/Man/Lau}

for proteins that interact with the environment. Again for nutrition researchers like yourself you need to include that in your equation. Copy number variation may account for some of the variation that we are seeing in these infants and children in response to different nutrition challenges.

Dr. Björkstén: The potential for personalized medicine is fascinating, but I would like to reiterate the ethical issues. It is clearly unethical to predict, unless you have treatment to offer.

\section{Reference}

1 Bergmann RL, Bergmann KE, Wahn U: Can we predict atopic disease using perinatal risk factors? Clin Exp Allergy 1998;28:905-907. 